\title{
Simulation of Multiphysics Multiscale Systems: Introduction to the ICCS'2007 Workshop
}

\author{
Valeria V. Krzhizhanovskaya ${ }^{1}$ and Shuyu Sun $^{2}$ \\ ${ }^{1}$ Section Computational Science, Faculty of Science, University of Amsterdam, \\ Kruislaan 403, 1098 SJ Amsterdam, The Netherlands \\ valeria@science.uva.nl \\ ${ }^{2}$ Department of Mathematical Sciences, Clemson University \\ O-221 Martin Hall, Clemson, SC 29634-0975, USA \\ shuyuaclemson. edu
}

\begin{abstract}
Modeling and simulation of multiphysics multiscale systems poses a grand challenge to computational science. To adequately simulate numerous intertwined processes characterized by different spatial and temporal scales (often spanning many orders of magnitude), sophisticated models and advanced computational techniques are required. The aim of the workshop on Simulation of Multiphysics Multiscale Systems (SMMS) is to facilitate the progress in this multidisciplinary research field. We provide an overview of the recent trends and latest developments, with special emphasis on the research projects selected for presentation at the SMMS'2007 workshop.
\end{abstract}

Keywords: Multiphysics, Multiscale, Complex systems, Modeling, Simulation, ICCS, Workshop.

\section{Introduction}

Real-life processes are inherently multiphysics and multiscale. From atoms to galaxies, from amino-acids to living organisms, nature builds systems that involve interactions amongst a wide range of physical phenomena operating at different spatial and temporal scales. Complex flows, fluid-structure interactions, plasma and chemical processes, thermo-mechanical and electromagnetic systems are just a few examples essential for fundamental and applied sciences. Numerical simulation of such complex multiscale phenomena is vital for better understanding Nature and for advancing modern technologies. Due to the tremendous complexity of multiphysics multiscale systems, adequate simulation requires development of sophisticated models and smart methods for coupling different scales and levels of description (nano-micro-mesomacro). Until recently, such coupled modeling has been computationally prohibitive. But spectacular advances in computer performance and emerging technologies of parallel distributed grid computing have provided the community of computational physicists with the tools to break the barriers and bring simulation to a higher level of detail and accuracy. On the other hand, this progress calls for new efficient numerical algorithms and advanced computational techniques specific to the field where coupling different models or scales within one simulation is essential. 
In the last decade, modeling and simulation showed a clear trend away from simplified models that treat the processes on a single scale toward advanced self-adapting multiscale and multi-model simulations. The importance of such advanced computer simulations is recognized by various research groups and supported by national and international projects, e.g. the Dutch Computational eScience Initiative [1], the SCaLeS initiative in the USA [2] and the Virtual Physiological Human EU project [3]. Many significant developments were accomplished as a result of joint efforts in the multidisciplinary research society of physicists, biologists, computational scientists and computer experts. To boost scientific cross-fertilization and promote collaboration of these diverse groups of specialists, we have launched a series of mini-symposia on Simulation of Multiphysics Multiscale Systems (SMMS) in conjunction with the International Conference on Computational Sciences (ICCS) [4].

The fourth workshop in this series, organized as part of ICCS'2007, expands the scope of the meeting from physics and engineering to biological and biomedical applications. This includes computational models of tissue- and organo-genesis, tumor growth, blood vessel formation and interaction with the hosting tissue, biochemical transport and signaling, biomedical simulations for surgical planning, etc. The topics traditionally addressed by the symposium include modeling of multiphysics and/or multiscale systems on different levels of description, novel approaches to combine different models and scales in one problem solution, advanced numerical methods for solving multiphysics multiscale problems, new algorithms for parallel distributed computing specific to the field, and challenging multiphysics multiscale applications from industry and academia.

A large collection of rigorously reviewed papers selected for the workshops highlights modern trends and recent achievements [5]. It shows in particular the progress made in coupling different models (such as continuous and discrete models; quantum and classical approaches; deterministic and stochastic techniques; nano, micro, meso and macro descriptions) and suggests various coupling approaches (e.g. homogenization techniques, multigrid and nested grids methods, variational multiscale methods; embedded, concurrent, integrated or hand-shaking multiscale methods, domain bridging methods, etc.). A selected number of papers have been published in the special issues of International Journal for Multiscale Computational Engineering [6-7], collecting state-of-the-art methods for multiscale multiphysics applications covering a large spectrum of topics such as multiphase flows, discharge plasmas, turbulent combustion, chemical vapor deposition, fluid-structure interaction, thermo-mechanical and magnetostrictive systems, and astrophysics simulation. In this paper we overview the latest developments in modeling and simulation of multiphysics multiscale systems exemplified by the research presented at the SMMS'2007 workshop.

\section{Overview of Work Presented in This Workshop}

The papers presented in this workshop cover state-of-the-art simulations of multiphysics multiscale problems; they represent ongoing research projects on various important topics relevant to the modeling and computation of these complex systems. Numerical simulations of these problems require two essential components. The first one is the development of sophisticated models for each physical process, 
characterized by its own specific scales and its own mechanisms, and integration of these models into one seamless simulation. Coupling or extension of atomistic and continuum models studied in [8-14] shows that sophisticated modeling is essential to accurately represent the physical world. Similarly, works in [15-18] demonstrate that biological or biomedical systems have intrinsically multiscale nature and require multiscale modeling. The second essential component for numerical simulation of multiphysics and multiscale problems includes efficient numerical algorithms and advanced computational techniques. Computational methodologies and programming tools [19-21] and advanced mathematical and numerical algorithms [22-25] are indispensable for efficient implementation of multiscale multiphysics models, which are computationally very intensive and often intractable using ordinary methods. Cellular automata [11,26,27] and the lattice Boltzmann method [28-31], which can be considered both as modeling tools and as numerical techniques, prove to be very powerful and promising in modeling complex flows and other multiscale complex systems.

The projects in [8-10] investigate computationally efficient yet physically meaningful ways of coupling discrete and continuum models across multiple scales. Another way of treating multiscale problems is to develop single-scale approximation models. Papers [12,13] present development and analysis of models at an atomic or molecular scale, while project [11] couples multiple continuum models at a macroscopic scale. In [8], an adaptively coupled approach is presented for compressible viscous flows, based on the overlapped Schwarz coupling procedure. The continuum domain is described by Navier-Stokes equations solved using a finite volume formulation in compressible form to capture the shock, and the molecular domain is solved by the Direct Simulation Monte Carlo method. Work conducted in [9] leads to development and application of two computational tools linking atomistic and continuum models of gaseous systems: the first tool, a Unified Flow Solver for rarefied and continuum flows, is based on a direct Boltzmann solver and kinetic CFD schemes, and the second tool is a multiscale computational environment integrating CFD tools with Kinetic Monte Carlo and Molecular Dynamics tools. Paper [10] describes an application of the Unified Flow Solver (UFS) for complex gas flows with rarefied and continuum regions. The UFS methodology is based on the direct numerical solution of the Boltzmann equation for rarefied flow domains and the kinetic schemes of gas dynamics (for the Euler or Navier-Stokes equations) for continuum flow domains.

In [13], molecular dynamics simulations are extended to slow dynamics that could arise in materials science, chemistry, physics and biology. In particular, the hyperdynamics method developed for low-dimension energy-dominated systems is extended to simulate slow dynamics in atomistic general systems. In [12], a new isothermal quantum Euler model is derived and the asymptotic behavior of the quantum Euler system is formally analyzed in the semiclassical and zero-temperature limits. To simulate the process of biomass conversion [14], a submodel is developed for reverse combustion process in a solid fuel layer on the grate. It gives good predictions for the velocity of combustion front and spatial profiles of porosity, oxygen fraction and temperature, which are essential inputs for $\mathrm{NO}_{\mathrm{x}}$ calculations.

Multiscale approaches proved to be very useful for modeling and simulation of biological and biomedical systems [15-18]. In [15], a multiscale cell-based model is presented that addresses three stages of cancer development: avascular tumor growth, tumor-induced angiogenesis, and vascular tumor growth. The model includes the 
following three levels that are integrated through a hybrid MPI parallel scheme: the intracellular regulations that are described by Boolean networks, the cellular level growth and dynamics that are described by a lattice Monte Carlo model, and the extracellular dynamics of the signaling molecules and metabolites that are described by a system of reaction-diffusion equations. The work [17] is related to the analysis of dynamics of semi-flexible polymers, such as DNA molecules. A new efficient approximate technique predicts material properties of the polymeric fluids accounting for internal viscosity. The results explain the phenomenon of shear thinning and provide better predictions compared to the traditional techniques. In [16], coupled autoregulated oscillators in a single- and multi-cellular environment are modeled, taking into consideration intrinsic noise effects in genetic regulation, characterized by delays due to the slow biochemical processes. Diverse disciplines including physiology, biomechanics, fluid mechanics and simulation are brought together in [18] to develop a predictive model of the behavior of a prosthetic heart valve by applying simulation techniques for the study of cardiovascular problems, such as blood clotting. A commercial finite volume computational fluid dynamics code ANSYS/CFX is used for the 3D components of the model.

Advanced mathematical and numerical algorithms are required for effective coupling of various models across multiple scales and for efficient reduction of the computations needed for fine scale simulations without loss of accuracy [22-25]. As a significant extension to the classical multiscale finite element methods, paper [24] is devoted to the development of a theoretical framework for multiscale Discontinuous Galerkin (DG) methods and their application to efficient solutions of flow and transport problems in porous media with interesting numerical examples. In this work, local DG basis functions at the coarse scale are first constructed to capture the local properties of the differential operator at the fine scale, and then the DG formulations using the newly constructed local basis functions instead of conventional polynomial functions are solved on the coarse scale elements. In [23], an efficient characteristic finite element method is proposed for solving the magnetic induction equation in magnetohydrodynamics, with numerical results exhibiting how the topological structure and energy of the magnetic field evolve for different resistivity scales. Paper [22] includes a fast Fourier spectral technique to simulate the Navier-Stokes equations with no-slip boundary conditions, enforced by an immersed boundary technique called volume-penalization. In [25], a deflation technique is proposed to accelerate the iterative processing of the linear system built from discretization of the pressure Poisson equation with bubbly flow problems.

A number of computational methodologies and programming tools have been developed for simulations of multiscale multiphysics systems [19-21]. In the mesh generation technique presented in [21], surface reconstruction in applications involving complex irregular domains is considered for modeling biological systems, and an efficient and relatively simple approach is proposed to automatically recover a high quality surface mesh from low-quality non-consistent inputs that are often obtained via 3-D acquisition systems like magnetic resonance imaging, microscopy or laser scanning. In [20], a new methodology for two-way connection of microscopic model and macroscopic model, called Macro-Micro Interlocked simulation, is presented for multiscale simulations, together with demonstration of the applicability of the methodology for the various phenomena, such as cloud formation in atmosphere, gas 
detonation, aurora, solid friction, and onset of solar flares. Paper [19] addresses the challenge arising from the intercomponent data exchanges among components of multiscale models and the language interoperability between their various constituent codes. This work leads to the creation of a set of interlanguage bindings for a successful parallel coupling library, the Model Coupling Toolkit.

Automaton, a mathematical model for a finite state machine, has been studied as a paradigm for modeling multiscale complex systems [11,26,27]. Systems considered in [26] arise from the modeling of weed dispersal. In this work, the systems are approximated by pathways through a network of cells, and the results of simulations provide evidence that the method is suitable for modeling weed propagation mechanisms using multiple scales of observation. In [27], complex automata are formalized with identification of five classes of scale separation and further investigation of the scale separation map in relation with its capability to specify its components. Efforts are spent in [11] on the application of macroscopic modeling with cellular automata to simulation of lava flows, which consist of unconfined multiphase streams, the characteristics of which vary in space and time as a consequence of many interrelated physical and chemical phenomena.

The lattice Boltzmann method, being a discrete computational method based upon the Boltzmann equation, is a powerful mesoscopic technique for modeling a wide variety of complex fluid flow problems. In addition to its capability to accommodate a variety of boundary conditions, this approach is able to bridge microscopic phenomena with the continuum macroscopic equations [28-31]. In [28], the problem of mixed convection in a driven cavity packed with porous medium is studied. A lattice Boltzmann model for incompressible flow in porous media and another thermal lattice Boltzmann model for solving energy equation are proposed based on the generalized volume-averaged flow model. Project [31] presents a model for molecular transport effects on double diffusive convection; in particular, this model is intended to access the impact of variable molecular transport effects on the heat and mass transfer in a horizontal shallow cavity due to natural convection of a binary fluid. In [29], a multiscale approach is applied to model the polymer dynamics in the presence of a fluid solvent, combining Langevin molecular dynamics techniques with a mesoscopic lattice Boltzmann method for the solvent dynamics. This work is applied in the interesting context of DNA translocation through a nanopore. In [30], the lattice Boltzmann method for convection-diffusion equation with source term is applied directly to solve some important nonlinear complex equations by using complex-valued distribution function and relaxation time.

\section{Conclusions}

The progress in understanding physical, chemical, biological, sociological and even economical processes is strongly dependent on adequacy and accuracy of numerical simulation. All the systems important for scientific and industrial applications are essentially multiphysics and multiscale: they are characterized by the interaction of a great number of intertwined processes that operate at different spatial and temporal scales. Modern simulation technologies make efforts to bridge the gaps between different levels of description, and to seamlessly combine the scales spanning many 
orders of magnitude in one simulation. The progress in developing multiphysics multiscale models and specific numerical methodologies is exemplified by the projects presented in the SMMS workshops [4].

Acknowledgments. We would like to thank the participants of our workshop for their inspiring contributions, and the members of the Program Committee for their diligent work, which led to the very high quality of the conference. Special thanks go to Alfons Hoekstra for his efficient and energetic work on preparing the SMMS'2007 workshop. The organization of this event was partly supported by the NWO/RFBR projects \# 047.016.007 and 047.016.018, and the Virtual Laboratory for e-Science Bsik project.

\section{References $^{*}$}

1. P.M.A. Sloot et al. White paper on Computational e-Science: Studying complex systems in silico. A National Research Initiative. December 2006 http://www.science.uva.nl/ research/pscs/papers/archive/Sloot2006d.pdf

2. SCaLeS: A Science Case for Large Scale Simulation: http://www.pnl.gov/scales/

3. N. Ayache et al. Towards Virtual Physiological Human: Multilevel modelling and simulation of the human anatomy and physiology. White paper. 2005.

http://ec.europa.eu/information_society/activities/health/docs/events/barcelona2005/ ec-vph-white-paper2005nov.pdf

4. http://www.science.uva.nl/ valeria/SMMS

5. LNCS V. 3992/2006 DOI 10.1007/11758525, pp. 1-138;

LNCS V. 3516/2005 DOI 10.1007/b136575, pp. 1-146;

LNCS V. 3039/2004 DOI 10.1007/b98005, pp. 540-678

6. V.V. Krzhizhanovskaya, B. Chopard, Y.E. Gorbachev (Eds.) Simulation of Multiphysics Multiscale Systems. Special Issue of International Journal for Multiscale Computational Engineering. V. 4, Issue 2, 2006. DOI: 10.1615/IntJMultCompEng.v4.i2

7. V.V. Krzhizhanovskaya, B. Chopard, Y.E. Gorbachev (Eds.) Simulation of Multiphysics Multiscale Systems. Special Issue of International Journal for Multiscale Computational Engineering. V. 4, Issue 3, 2006. DOI: 10.1615/IntJMultCompEng.v4.i3

8. G. Abbate, B.J. Thijsse, and C.R.K. Kleijn. Coupled Navier-Stokes/DSMC for transient and steady-state flows. Proceedings ICCS'2007.

9. V.I. Kolobov, R.R. Arslanbekov, and A.V. Vasenkov. Coupling atomistic and continuum models for multi-scale simulations. Proceedings ICCS'2007.

10. V.V. Aristov, A.A. Frolova, S.A. Zabelok, V.I. Kolobov, R.R. Arslanbekov. Simulations of multiscale gas flows on the basis of the Unified Flow Solver. Proceedings ICCS'2007.

11. M.V. Avolio, D. D’Ambrosio, S. Di Gregorio, W. Spataro, and R. Rongo. Modelling macroscopic phenomena with cellular automata and parallel genetic algorithms: an application to lava flows. Proceedings ICCS'2007.

12. S. Gallego, P. Degond, and F. Mhats. On a new isothermal quantum Euler model: Derivation, asymptotic analysis and simulation. Proceedings ICCS'2007.

* All papers in Proceedings of ICCS'2007 are presented in the same LNCS volume, following this paper. 
13. X. Zhou and Y. Jiang. A general long-time molecular dynamics scheme in atomistic systems. Proceedings ICCS'2007.

14. R.J.M. Bastiaans, J.A. van Oijen, and L.P.H. de Goey. A model for the conversion of a porous fuel bed of biomass. Proceedings ICCS'2007.

15. Y. Jiang. Multiscale, cell-based model for cancer development. Proceedings ICCS'2007.

16. A. Leier and P. Burrage. Stochastic modelling and simulation of coupled autoregulated oscillators in a multicellular environment: the her $1 /$ her7 genes. Proceedings ICCS'2007.

17. J. Yang and R.V.N. Melnik. A new model for the analysis of semi-flexible polymers with internal viscosity and applications. Proceedings ICCS'2007.

18. V. Diaz Zuccarini. Multi-physics and multiscale modelling in cardiovascular physiology: Advanced users methods of biological systems with CFX. Proceedings ICCS'2007.

19. E.T. Ong et al. Multilingual interfaces for parallel coupling in multiphysics and multiscale systems. Proceedings ICCS'2007.

20. K. Kusano, A. Kawano, and H. Hasegawa. Macro-micro interlocked simulations for multiscale phenomena. Proceedings ICCS'2007.

21. D. Szczerba, R. McGregor, and G. Szekely. High quality surface mesh generation for multi-physics bio-medical simulations. Proceedings ICCS'2007.

22. G.H. Keetels, H.J.H. Clercx, and G.J.F. van Heijst. A Fourier spectral solver for wall bounded 2D flow using volume-penalization. Proceedings ICCS'2007.

23. J. Liu. An efficient characteristic method for the magnetic induction equation with various resistivity scales. Proceedings ICCS'2007.

24. S. Sun. Multiscale discontinuous Galerkin methods for modeling flow and transport in porous media. Proceedings ICCS'2007.

25. J.M. Tang and C. Vuik. Acceleration of preconditioned Krylov solvers for bubbly flow problems. Proceedings ICCS'2007.

26. A.G. Dunn and J.D. Majer. Simulating weed propagation via hierarchical, patch-based cellular automata. Proceedings ICCS'2007.

27. A.G. Hoekstra. Towards a complex automata framework for multi-scale modelling formalism and the scale separation map. Proceedings ICCS'2007.

28. Z. Chai, B. Shi, and Z. Guo. Lattice Boltzmann simulation of mixed convection in a driven cavity packed with porous medium. Proceedings ICCS'2007.

29. S. Melchionna, E. Kaxiras, and S. Succi. Multiscale modeling of biopolymer translocation through a nanopore. Proceedings ICCS'2007.

30. B.C. Shi. Lattice Boltzmann simulation of some nonlinear complex equations. Proceedings ICCS'2007.

31. X.M. Yu, B.C. Shi, and Z.L. Guo. Numerical study of molecular transport effects on double diffusive convection with lattice Boltzmann method. Proceedings ICCS'2007. 